\title{
Sessile Serrated Adenoma / Polyp (SSA/P) Carcinogenic Mechanism and Gene Mutations Caused by Fusobacterium Nucleatum - Problems to be Clarified in the Future
}

\author{
Tadashi Ohara* \\ Department of Gastroenterology, Kitashinagawa Third Hospital, Japan \\ *Corresponding author: Tadashi Ohara, Department of Gastroenterology, Kitashinagawa Third Hospital, Kohno Clinical Medicine \\ Research Institute, 3-3-7 Kitashinagawa, Shinagawa, Tokyo 140-0001, Japan.
}

To Cite This Article: Tadashi Ohara, Sessile Serrated Adenoma / Polyp (SSA/P) Carcinogenic Mechanism and Gene Mutations Caused by Fusobacterium Nucleatum - Problems to be Clarified in the Future . Am J Biomed Sci \& Res. 2021 - 12(1). AJBSR.MS.ID.001702.

DOI: 10.34297/AJBSR.2021.12.001702.

Received: 㠿 February 09, 2021; Published: 笽 February 16, 2021

\section{Introduction}

The carcinogenic pathways of colorectal cancer include the classical pathway in which adenoma grows to colorectal cancer and the serrated pathway in which colorectal cancer grows from serrated adenoma. In the former adenoma, the diversity of gut flora ( $\alpha$ diversity) is reduced, but not in SSA/P, and there is a difference in the change of gut flora depending on the carcinogenic route [1]. The serrated pathway is a concept recently proposed by Torlakovic et al. [2]. Sessile serrated adenoma / polyp (SSA/P) predominates in the right-side colon [3] and SSA/P is caused by mutations in the BRAF gene. In SSA/P, DNA methylation is observed genome-wide, and p16 and IGFBP7 are methylated to shift cells from the resting phase to the proliferative phase [4]. In addition, the methylation of the mismatch repair gene MLH1 suppresses MLH1 protein expression, causing microsatellite instability (MSI), resulting in atypia from SSA/P. Tumors caused by this atypia are called SSA/P with cytological dysplasia [5], and this concept was also proposed at WHO in 2010. The initial SSA/P lesion, which is the origin of colorectal cancer, has a flat, white-like color tone, is difficult to distinguish endoscopically from normal mucosa, and is often overlooked. Inaccurate endoscopic observations of SSA/P miss the detection of early-stage colorectal carcinoma [6]. The accurately diagnose SSA/P endoscopically is a future clinical issue for colorectal cancer. We have great expectations for endoscopic image analysis systems that have made remarkable progress in recent years.

Fusobacterium nucleatum is known to induce gene mutations. In Fusobacterium nucleatum-infected colonic mucosal tissue, CpG island methylator phenotype-high (CIMP-high), MSI positive, and MLH1 hypermethylation are observed (24). In addition, there is an interesting report that SSA/P is more likely to be Fusobacterium genus-positive as the site of origin goes from the sigmoid colon to the ascending colon to the cecum to the oral-side deep colon [7]. This is not seen in classical colorectal carcinogenesis pathway. Large amounts of Fusobacterium nucleatum have also been reported to be associated with MSI-high, MLH1 hypermethylated CIMP-high, and BRAF mutations [8]. These reports are very interesting. Tumor immunity may be avoided in MSI-high colorectal cancer [9]. However, not all Fusobacterium nucleatum-infected and MSI-high mucosal tissues grow into cancerous, and it is thought that undiscovered factors and events that trigger carcinogenesis are hidden, and the details of carcinogenesis are still unclear. We would like to pay attention to future research on the details of gene mutations caused by Fusobacterium nucleatum, SSA/P carcinogenesis and BRAF gene mutations.

\section{Conflict of Interest}

None declared.

\section{References}

1. Peters BA, Dominianni C, Shapiro JA, Church TR, Wu J, et al. (2016) The gut microbiota in conventional and serrated precursors of colorectal cancer. Microbiome 4(1): 69.

2. Torlakovic E, Snover DC (1996) Serrated adenomatous polyposis in humans. Gastroenterol 110(3): 748-755. 
3. Ban S, Mitomi H, Horiguchi H, Sato H, Shimizu M (2014) Adenocarcinoma arising in small sessile serrated adenoma/polyp (SSA/P) of the colon: clinicopathological study of eight lesions. Pathol Int 64(3): 123-132.

4. Kaji E, Uraoka T, Kato J, Hiraoka S, Suzuki H, et al. (2012) Externalization of saw-tooth architecture in small serrated polyps implies the presence of methylation of IGFBP7. Dig Dis Sci 57(5): 1261-1270.

5. Matthew FK (2013) Sessile serrated polyps: an important route to colorectal cancer. J Natl Compr Cnnc Netw 11(12): 1586-1594.

6. Rashtak S, Rego R, Sweetser SR, Sinicrope FA (2017) Sessile serried polyps and colon cancer prevention. Cancer Prev Res (Phila) 10(5): 270278.
7. Tahara t, Yamamoto E, Suzuki H, Maruyama R, Chung W, et al. (2014) Fusobacterium in colonic flora and molecular features of colorectal carcinoma. Cancer Res 74(5): 1311-1318.

8. Mima K, Nishihara R, Qian ZR, Cao Y, Sukawa Y, et al. (2016) Fusobacterium nucleatum in colorectal carcinoma tissue and patient prognosis. Gut 65(12): 1973-1980.

9. Hamada T, Zhang X, Mima K, Bullman S, Sukawa Y, et al. (2018) Fusobacterium nucleatum in colorectal cancer relate to immune response differentially by tumor microsatellite instability status. Cancer Immunol Res 6(11): 1327-1336. 